\title{
On weak-strong uniqueness property for the full compressible magnetohydrodynamics flows
}

\author{
Weiping Yan
}

Received: date / Accepted: date

\begin{abstract}
This paper is devoted to the study of the weak-strong uniqueness property for the full compressible magnetohydrodynamics flows. The governing equations for magnetohydrodynamic flows are expressed by the full NavierStokes system for compressible fluids enhanced by forces due to the presence of the magnetic field as well as the gravity and with an additional equation which describes the evolution of the magnetic field. Using the relative entropy inequality, we prove that a weak solution coincides with the strong solution, emanating from the same initial data, as long as the latter exists.
\end{abstract}

Keywords magnetohydrodynamic flows · weak solution · strong solution · entropy

Mathematics Subject Classification (2010) 76W05 - 35D30 - 35D35 and $54 \mathrm{C} 70$

\section{Introduction and Main results}

This paper studies the weak-strong uniqueness property of the viscous compressible magnetohydrodynamic flows

$$
\begin{aligned}
& \partial_{t} \rho+\operatorname{div}_{x}(\rho \mathbf{u})=0, \\
& \partial_{t}(\rho \mathbf{u})+\operatorname{div}_{x}(\rho \mathbf{u} \otimes \mathbf{u})+\nabla_{x} P(\rho, \theta)=\operatorname{div}_{x} \mathbf{S}+\mathbf{J} \times \mathbf{H}, \\
& \partial_{t}(\rho s(\rho, \theta))+\operatorname{div}_{x}(\rho s(\rho, \theta) \mathbf{u})+\operatorname{div}_{x}\left(\frac{\mathbf{q}}{\theta}\right)=\sigma \\
& \partial_{t} \mathbf{H}-\nabla \times(\mathbf{u} \times \mathbf{H})+\nabla \times(\nu \nabla \times \mathbf{H})=0
\end{aligned}
$$

College of of Mathematics, Jilin University, Changchun 130012, P.R. China.

Beijing International Center for Mathematical Research, Peking University, Beijing 100871, P.R. China.

E-mail: yan8441@126.com 
where $\mathbf{u}$ is the vector field, $\rho$ is the density, $\theta$ is the temperature, $\mathbf{J}$ is the electronic current, $e(\rho, \theta)$ is the (specific) internal energy and $\mathbf{H}$ is the magnetic field. The electronic current satisfies Ampère's law

$$
\mathbf{J}=\nabla \times \mathbf{H}
$$

whereas the Lorentz force is given by

$$
\mathbf{J} \times \mathbf{H}=\operatorname{div}_{x}\left(\frac{1}{\mu} \mathbf{H} \otimes \mathbf{H}-\frac{1}{2 \mu}|\mathbf{H}|^{2} \mathbf{I}\right),
$$

with $\mu$ being a permeability constant of free space, which here is assumed to be $\mu=1$ for simplicity of the presentation. The electronic current $\mathbf{J}$, the electric field $\mathbf{E}$ and the magnetic field $\mathbf{H}$ are related through Ohm's law

$$
\mathbf{J}=\sigma(\mathbf{E}+\mathbf{u} \times \mathbf{H}) .
$$

The interaction described by the theory of magnetohydrodynamics, "collective effects," is governed by the Faraday's law,

$$
\partial_{t} \mathbf{H}+\nabla \times \mathbf{E}=0, \quad \operatorname{div}_{x} \mathbf{H}=0 .
$$

Taking into consideration (7) we are able to write (8) in the following form

$$
\partial_{t} \mathbf{H}+\nabla \times(\mathbf{H} \times \mathbf{u})+\nabla \times(\nu \nabla \times \mathbf{H})=0,
$$

where $\nu=\frac{1}{\sigma}$.

Motivated by several recent studies devoted to the scale analysis as well as numerical experiments related to the proposed model (see Klein et al. [13]), we suppose that the viscous stress $\mathbf{S}$ is a linear function of the velocity gradient, therefore described by Newton's law

$$
\mathbf{S}\left(\theta, \nabla_{x} \mathbf{u}\right)=\mu(\theta)\left(\nabla_{x} \mathbf{u}+\nabla_{x}^{\perp} \mathbf{u}-\frac{2}{3} \operatorname{div}_{x} \mathbf{u I}\right)+\eta(\theta) \operatorname{div}_{x} \mathbf{u I},
$$

while $\mathbf{q}$ is the heat flux satisfying Fourier's law

$$
\mathbf{q}=-\kappa(\theta) \nabla_{x} \theta,
$$

and $\sigma$ stands for the entropy production rate which is non-negative measure given by

$$
\sigma \geq \frac{1}{\theta}\left(\mathbf{S}\left(\theta, \nabla_{x} \mathbf{u}\right): \nabla_{x} \mathbf{u}-\frac{\mathbf{q}\left(\theta, \nabla_{x} \theta\right) \cdot \nabla_{x} \theta}{\theta}\right) .
$$

We supplement compressible magnetohydrodynamic flows (11)-(14) with conservation boundary condition:

$$
\left.\mathbf{u}\right|_{\partial \Omega}=\left.\mathbf{q} \cdot \mathbf{n}\right|_{\partial \Omega}=0,
$$

and

$$
\left.\mathbf{H}\right|_{\partial \Omega}=0
$$


The concept of weak solution in fluid dynamics was introduced by Leray [16] in the context of incompressible, linearly viscous fluids. The original ideas of Leray have been put into the elegant framework of generalized derivatives (distributions) and the associated abstract function spaces of Sobolev type (For example, see Ladyzhenskaya [15] and Temam [22]). Lions [17] extended the theory to the class of barotropic flows (see also [6]). One of meaningful compressible flow models is the compressible magnetohydrodynamics (MHD). It is a combination of the compressible Navier-Stokes equations of fluid dynamics and Maxwell's equations of electromagnetism. Ducomet and Feireisl 4 proved that the existence of global in time weak solutions to a multi-dimensional nonisentropic MHD system for gaseous stars coupled with the Poisson equation with all the viscosity coefficients and the pressure depending on temperature and density asymptotically, respectively. Hu and Wang [11] studied the global variational weak solution to the three-dimensional full magnetohydrodynamic equations with large data by an approximation scheme and a weak convergence method. Jiang, et all. 12] obtained that the convergence towards the strong solution of the ideal incompressible MHD system in the periodic domains. Recently, Kwon, et all [14] established the incompressible limits of weak solutions to the compressible magnetohydrodynamics flows (1)-(4) on both bounded and unbounded domains.

The physical properties of the magnetohydrodynamics flows are reflected through various constitutive relations which are expressed as typically nonlinear functions relating the pressure $P=P(\rho, \theta)$, the internal energy $e(\rho, \theta)$, the specific entropy $s=s(\rho, \theta)$ to the macroscopic variables $\rho$, $\mathbf{u}$, and $\theta$. According to the fundamental principles of thermodynamics, the specific internal energy $e$ is related to the pressure $P$, and the specific entropy s through Gibbs' relation

$$
\theta D s(\rho, \theta)=D e(\rho, \theta)+P(\rho, \theta) D\left(\frac{1}{\rho}\right),
$$

where $D$ denotes the differential with respect to the state variables $\rho$ and $\theta$.

Since the lack of information resulting from the inequality sign in (12), we need supplement the resulting system with the energy inequality,

$$
\frac{d}{d t} \int_{\Omega}\left(\frac{1}{2} \rho|\mathbf{u}|^{2}+\rho e(\rho, \theta)+\frac{1}{2}|\mathbf{H}|^{2}\right) d x+\int_{\Omega}\left(|\nabla \mathbf{u}|^{2}+\nu|\nabla \times \mathbf{H}|^{2}\right) \leq 0 .
$$

Thus the total energy $\mathcal{E}$ is given by

$$
\mathcal{E}=\frac{1}{2} \rho|\mathbf{u}|^{2}+\rho e(\rho, \theta)+\frac{1}{2}|\mathbf{H}|^{2} .
$$

Under these circumstances, it can be shown (see [7, Chapter 2) that any weak solution of (1) that is sufficiently smooth satisfies, instead of (12), the standard relation

$$
\sigma=\frac{1}{\theta}\left(\mathbf{S}\left(\theta, \nabla_{x} \mathbf{u}\right): \nabla_{x} \mathbf{u}-\frac{\mathbf{q}\left(\theta, \nabla_{x} \theta\right) \cdot \nabla_{x} \theta}{\theta}\right) .
$$


The pressure $P=P(\rho, \theta)$ is here expressed as

$$
P=P_{F}+P_{R}, \quad P_{R}=\frac{a}{3} \theta^{4}, a>0,
$$

where $P_{R}$ denotes the radiation pressure. Moreover, we shall assume that $P_{F}=$ $P_{M}+P_{E}$, where $P_{M}$ is the classical molecular pressure obeying Boyle's law, while $P_{E}$ is the pressure of electron gas constituent behaving like a Fermi gas in the degenerate regime of high densities and/or low temperatures (see Chapters 1, 15 in Eliezer et al. [5]). Thus necessarily $P_{F}$ takes the form

$$
P_{F}=\theta^{\frac{5}{2}} p\left(\frac{\rho}{\theta^{\frac{3}{2}}}\right),
$$

where $p \in \mathbf{C}^{1}[0, \infty)$ satisfies

$$
p(0)=0, \quad p^{\prime}(Z)>0 \text { for all } z \geq 0 .
$$

In agreement with Gibbs' relation (15), the internal energy can be taken as

$$
e=e_{F}+e_{R}, \text { with } e_{R}=a \frac{\theta^{4}}{\rho},
$$

where $e_{F}=e_{F}(\rho, \theta), P_{F}(\rho, \theta)$ are interrelated through the following equation of state

$$
P_{F}(\rho, \theta)=\frac{2}{3} \rho e_{F}(\rho, \theta) .
$$

We need the thermodynamic stability hypothesis:

$$
\frac{\partial P(\rho, \theta)}{\partial \rho}>0, \frac{\partial e(\rho, \theta)}{\partial \theta}>0 \text { for all } \rho, \theta>0 .
$$

The second inequality in thermodynamic stability hypothesis (24) gives that

$$
0<\frac{\frac{5}{3} p(Z)-p^{\prime}(Z) Z}{Z}<c \text { for all } Z>0,
$$

which implies that the function $Z \mapsto \frac{p(Z)}{Z^{\frac{5}{3}}}$ is decreasing and we suppose that

$$
\lim _{Z \longrightarrow \infty} \frac{p(Z)}{Z^{\frac{5}{3}}}=p_{\infty}>0 .
$$

In accordance with (15) and (22) we set the entropy as

$$
s=s_{F}+s_{R}, \quad w t i h s_{F}=S\left(\frac{\rho}{\theta^{\frac{3}{2}}}\right), \quad s_{R}=\frac{4 a}{3 \rho} \theta^{3} .
$$

Furthermore, by the Third law of thermodynamics,

$$
S^{\prime}(Z)=-\frac{3}{2} \frac{\frac{5}{3} p(Z)-p^{\prime}(Z) Z}{Z^{2}}<0, \quad \lim _{Z \longrightarrow \infty} S(Z)=0 .
$$


We choose the transport coefficients in the form

$$
\begin{aligned}
& \mu(\theta)=\mu_{0}+\mu_{1} \theta, \quad \mu_{0}, \mu_{1}>0, \quad \eta \equiv 0, \\
& \kappa(\theta)=\kappa_{0}+\kappa_{2} \theta^{2}+\kappa_{3} \theta^{3}, \quad \kappa_{i}>0, \quad i=0,2,3 .
\end{aligned}
$$

A fundamental test of admissibility of a class of weak solutions to a given evolutionary problem is the property of weak-strong uniqueness. More specifically, the weak solution must coincide with a (hypothetical) strong solution emanating from the same initial data as long as the latter exists. This problem has been intensively studied for the incompressible Navier-Stokes system, for example, see 2, 18, 21. It is a bit more delicate in the case of compressible cases. The weak- strong uniqueness of compressible barotropic Navier-Stokes system and isentropic compressible Navier-Stokes system were established in [8, 9] and [19, respectively. P. Germain [19] provides only a partial and conditional answer to the weak-strong uniqueness problem for the compressible Navier-Stokes equations. This question is definitely solved in 9. More recently, Feireisl and Novotný [10] extended the problem to compressible Navier-StokesFourier system by the relative entropy inequality. The relative entropy in 10 is reminiscent to C.M. Dafermos [3] (who introduced the relatives entropies via the entropy flux pairs for the conservation laws), but is different from the C.M. Dafermos concept (in contrast to 3], it is based on the thermodynamic stability conditions).

Inspired by the work of Feireisl and Novotný [10], we prove that the weakstrong uniqueness of compressible three-dimensional magnetohydrodynamic equations. Our contribution is to construct suitable relative entropy inequality to (1)-(3). Then we overcome the presence of the magnetic field and its interaction with the hydrodynamic motion in the MHD flow of large oscillation.

We organize the rest of this paper as follows. In section 2 , we recall the definition of the weak solutions and strong solutions to the magnetohydrodynamic flows on bounded domains. Meanwhile, the relative entropy inequality of (11)-(4) is derived. In the last section, we give the rigorous proof of the weakstrong uniqueness property for the compressible magnetohydrodynamic flows on bounded domains in the spirit of Feireisl and Novotný [10].

\section{Relative entropy and Main result}

Let $\Omega \subset \mathbf{R}^{3}$ be a bounded Lipschitz domain. We recall the definition of weak solution for (1)-(4).

Definition 1 We say that a quantity $(\rho, \mathbf{u}, \theta, \mathbf{H})$ is a weak solution of the full magnetohydrodynamic flows (MHD) (1)-(4) supplemented with the initial data $\left(\rho_{0}, \mathbf{u}_{0}, s\left(\rho_{0}, \theta_{0}\right), \mathbf{H}_{0}\right)$, and $\rho_{0} \geq 0, \theta_{0}>0$ provided that the following holds.

i) The density $\rho$ is a non-negative function, $\rho \in \mathbf{C}_{\text {weak }}\left([0, T] ; \mathbf{L}^{\frac{5}{3}}(\Omega)\right)$, the velocity field $\mathbf{u} \in \mathbf{L}^{2}\left(0, T ; \mathbf{W}_{0}^{1,2}\left(\Omega ; \mathbf{R}^{3}\right)\right), \rho \mathbf{u} \in \mathbf{C}_{\text {weak }}\left([0, T] ; \mathbf{L}^{\frac{5}{4}}\left(\Omega ; \mathbf{R}^{3}\right)\right)$. 
Equation (1) is replaced by a family of integral identities

$$
\int_{\Omega} \rho(\tau, \cdot) \varphi(\tau, \cdot) d x-\int_{\Omega} \rho_{0} \varphi(0, \cdot) d x=\int_{0}^{\tau} \int_{\Omega}\left(\rho \partial_{t} \varphi+\rho \mathbf{u} \cdot \nabla_{x} \varphi\right) d x d t
$$

for any $\varphi \in \mathbf{C}^{1}([0, T] \times \bar{\Omega})$, and any $\tau \in[0, T]$.

ii) The balance of momentum holds in distributional sense, namely

$$
\begin{aligned}
& \int_{\Omega} \rho \mathbf{u}(\tau, \cdot) \cdot \varphi(\tau, \cdot) d x-\int_{\Omega} \rho_{0} \mathbf{u}_{0} \cdot \varphi(0, \cdot) d x \\
= & \int_{0}^{\tau} \int_{\Omega}\left(\rho \mathbf{u} \cdot \partial_{t} \varphi+\rho \mathbf{u} \otimes \mathbf{u}: \nabla_{x} \varphi+P \operatorname{div}_{x} \varphi-\mathbf{S}: \nabla_{x} \varphi\right. \\
& +[(\nabla \times \mathbf{H}) \times \mathbf{H}] \cdot \varphi) d x d t
\end{aligned}
$$

for any $\varphi \in \mathbf{C}^{1}\left([0, T] \times \bar{\Omega} ; \mathbf{R}^{3}\right),\left.\varphi\right|_{\partial \Omega}=0$ and any $\tau \in[0, T]$.

iii) The entropy balance (3) and (12) are replaced by a family of integral inequalities

$$
\begin{aligned}
& \int_{\Omega} \rho s\left(\rho_{0}, \theta_{0}\right) \varphi(0, \cdot) d x-\int_{\Omega} \rho s(\rho, \theta)(\tau, \cdot) \varphi(\tau, \cdot) d x \\
+ & \int_{0}^{\tau} \int_{\Omega}\left(\frac{\varphi}{\theta}\left(\mathbf{S}: \nabla_{x} \mathbf{u}-\frac{\mathbf{q} \cdot \nabla_{x} \theta}{\theta}\right) d x d t\right. \\
\leq & -\int_{0}^{\tau} \int_{\Omega}\left(\rho s(\rho, \theta) \partial_{t} \varphi+\rho s(\rho, \theta) \mathbf{u} \cdot \nabla_{x} \varphi+\frac{\mathbf{q} \cdot \nabla_{x} \varphi}{\theta}\right) d x d t
\end{aligned}
$$

for any $\varphi \in \mathbf{C}^{1}([0, T] \times \bar{\Omega}), \varphi \geq 0$ and almost all $\tau \in[0, T]$. Here the quantities $\mathbf{S}$ and $\mathbf{q}$ are given through the constitutive equations (10) and (11). Moreover, similarly to the above, all quantities must be at least integrable on $(0, T) \times \Omega$. In particular, $\theta$ belongs to $\mathbf{L}^{\infty}\left(0, T ; \mathbf{L}^{4}(\Omega)\right) \cap \mathbf{L}^{2}\left(0, T ; \mathbf{W}^{1,2}(\Omega)\right)$. In addition, we require $\theta$ to be positive for almost all $(t, x) \in(0, T) \times \Omega$.

iv) The total energy of the system satisfies the following inequality

$$
\begin{aligned}
\int_{\Omega}\left(\frac{1}{2} \rho|\mathbf{u}|^{2}+\rho e(\rho, \theta)+\frac{1}{2}|\mathbf{H}|^{2}\right) d x & +\int_{0}^{\tau} \int_{\Omega}\left(|\nabla \mathbf{u}|^{2}+\nu|\nabla \times \mathbf{H}|^{2}\right) d x d t \\
& \leq \int_{\Omega}\left(\frac{1}{2} \rho_{0}\left|\mathbf{u}_{0}\right|^{2}+\rho_{0} e\left(\rho_{0}, \theta_{0}\right)+\frac{1}{2}\left|\mathbf{H}_{0}\right|^{2}\right) d x
\end{aligned}
$$

for almost all $\tau \in[0, T]$.

v) The magnetic field $\mathbf{H} \in \mathbf{L}^{2}\left(0, T ; \mathbf{W}^{1,2}\left(\Omega ; \mathbf{R}^{3}\right)\right)$. The Maxwell equation (44) verifies

$$
\begin{aligned}
\int_{\Omega} \mathbf{H}(\tau, \cdot) \varphi(\tau, \cdot) d x & -\int_{\Omega} \mathbf{H}_{0} \varphi_{0} d x \\
& =\int_{0}^{\tau} \int_{\Omega}\left(\mathbf{H} \cdot \partial_{t} \varphi-(\mathbf{H} \times \mathbf{u}+\nu \nabla \times \mathbf{H}) \cdot(\nabla \times \varphi)\right) d x d t,
\end{aligned}
$$

where $\varphi \in \mathbf{C}^{1}\left([0, T] \times \bar{\Omega} ; \mathbf{R}^{3}\right),\left.\varphi\right|_{\partial \Omega}=0$ and any $\tau \in[0, T]$. 
The definition of strong solution is

Definition 2 We say that $\left(\rho^{\prime}, \mathbf{u}^{\prime}, \theta^{\prime}, \mathbf{H}^{\prime}\right)$ is a classical (strong) solution to the full magnetohydrodynamic system (1)-(4) in $(0, T) \times \Omega$ if

$$
\begin{aligned}
& \rho^{\prime} \in \mathbf{C}^{1}([0, T] \times \bar{\Omega}), \quad \theta^{\prime}, \partial_{t} \theta^{\prime}, \nabla^{2} \theta^{\prime} \in \mathbf{C}([0, T] \times \Omega), \\
& \mathbf{u}^{\prime}, \partial_{t} \mathbf{u}^{\prime}, \nabla^{2} \mathbf{u}^{\prime} \in \mathbf{C}\left([0, T] \times \Omega ; \mathbf{R}^{3}\right), \quad \mathbf{H}^{\prime}, \partial_{t} \mathbf{H}^{\prime}, \nabla^{2} \mathbf{H}^{\prime} \in \mathbf{C}\left([0, T] \times \Omega ; \mathbf{R}^{3}\right), \\
& \rho^{\prime}(t, x) \geq \rho>0, \quad \theta^{\prime}(t, x) \geq \theta_{0}^{\prime}>0, \quad \text { for all }(t, x),
\end{aligned}
$$

and $\rho^{\prime}, \mathbf{u}^{\prime}, \theta^{\prime}, \mathbf{H}^{\prime}$ satisfy equations (1)-(4), (18), together with the boundary conditions (13)-(14). Observe that hypothesis (36) implies the following regularity properties of the initial data:

$$
\begin{aligned}
& \rho(0)=\rho_{0} \in \mathbf{C}^{1}(\bar{\Omega}), \quad \rho_{0} \geq \rho_{0}^{\prime}>0 \\
& \mathbf{u}(0)=\mathbf{u}_{0} \in \mathbf{C}^{2}(\bar{\Omega}), \\
& \theta(0)=\theta_{0} \in \mathbf{C}^{2}(\bar{\Omega}), \quad \theta_{0} \geq \theta_{0}^{\prime}>0 \\
& \mathbf{H}(0)=\mathbf{H}_{0} \in \mathbf{C}^{2}(\bar{\Omega}) .
\end{aligned}
$$

Before giving the main result, we deduce a relative entropy inequality which is satisfied by any weak solution to the full magnetohydrodynamic system (1)(4).

Let $\{A, B, C, D\}$ be a quantity of smooth function, $A$ and $C$ bounded below away from zero in $[0, T] \times \Omega$, and $\left.B\right|_{\partial \Omega}=\left.D\right|_{\partial \Omega}=0$. Moreover, we assume that smooth functions $B$ and $D$ satisfy that

$$
\partial_{t} D-\nabla \times(B \times D)+\nabla \times(\nu \nabla \times D)=0 .
$$

Taking $\varphi=\frac{1}{2}|B|^{2}, \varphi=B$ and $\varphi=C>0$ as a test function in (31), (32) and the entropy inequality (33), respectively, we get

$$
\begin{aligned}
\int_{\Omega} \frac{1}{2} \rho|B|^{2}(\tau, \cdot) d x-\int_{\Omega} \frac{1}{2} \rho_{0}|B|^{2}(0, \cdot) d x= & \int_{0}^{\tau} \int_{\Omega}\left(\rho B \cdot \partial_{t} B+\rho \mathbf{u} \cdot \nabla_{x} B \cdot B\right) d x d t,(39) \\
\int_{\Omega} \rho \mathbf{u} \cdot B(\tau, \cdot) d x-\int_{\Omega} \rho_{0} \mathbf{u}_{0} \cdot B(0, \cdot) d x= & \int_{0}^{\tau} \int_{\Omega}\left(\rho \mathbf{u} \cdot \partial_{t} B+\rho \mathbf{u} \otimes \mathbf{u}: \nabla_{x} B+P(\rho, \theta) \operatorname{div}_{x} B\right. \\
& \left.-\mathbf{S}\left(\theta, \nabla_{x} \mathbf{u}\right): \nabla_{x} B+((\nabla \times \mathbf{H}) \times \mathbf{H}) \cdot B\right) d x d t(40)
\end{aligned}
$$

and

$$
\begin{aligned}
\int_{\Omega} \rho_{0} s\left(\rho_{0}, \theta_{0}\right) C(0, \cdot) d x & -\int_{\Omega} \rho s(\rho, \theta) C(\tau, \cdot) d x+\int_{0}^{\tau} \int_{\Omega} \frac{C}{\theta}\left(\mathbf{S}\left(\theta, \nabla_{x} \mathbf{u}\right): \nabla_{x} \mathbf{u}-\frac{\mathbf{q}\left(\theta, \nabla_{x} \theta\right) \cdot \nabla_{x} \theta}{\theta}\right) d x d t \\
& \leq-\int_{0}^{\tau} \int_{\Omega}\left(\rho s(\rho, \theta) \partial_{t} C+\rho s(\rho, \theta) \mathbf{u} \cdot \nabla_{x} C+\frac{\mathbf{q}\left(\theta, \nabla_{x} \theta\right)}{\theta} \cdot \nabla_{x} C\right) d x d t
\end{aligned}
$$


It follows from (39), (40) and the energy inequality (34) that

$$
\begin{aligned}
\int_{\Omega}\left(\frac{1}{2} \rho|\mathbf{u}-B|^{2}+\right. & \left.\rho e(\rho, \theta)+\frac{1}{2}|\mathbf{H}|^{2}\right)(\tau, \cdot) d x+\int_{0}^{\tau} \int_{\Omega}\left(|\nabla \mathbf{u}|^{2}+\nu|\nabla \times \mathbf{H}|^{2}\right) d x d t \\
\leq & \int_{\Omega}\left(\frac{1}{2} \rho_{0}\left|\mathbf{u}_{0}-B(0, \cdot)\right|^{2}+\rho_{0} e\left(\rho_{0}, \theta_{0}\right)+\frac{1}{2}\left|\mathbf{H}_{0}\right|^{2}\right) d x \\
& +\int_{0}^{\tau} \int_{\Omega}\left(\left(\rho \partial_{t} B+\rho \mathbf{u} \cdot \nabla_{x} B\right) \cdot(B-\mathbf{u})-P(\rho, \theta) \operatorname{div}_{x} B\right. \\
& \left.+\mathbf{S}\left(\theta, \nabla_{x} \mathbf{u}\right): \nabla_{x} B-((\nabla \times \mathbf{H}) \times \mathbf{H}) \cdot B\right) d x d t .
\end{aligned}
$$

Then summing up (41) and (42), we deduce that

$$
\begin{aligned}
& \int_{\Omega}\left(\frac{1}{2} \rho|\mathbf{u}-B|^{2}+\rho e(\rho, \theta)+\frac{1}{2}|\mathbf{H}|^{2}-C \rho s(\rho, \theta)\right)(\tau, \cdot) d x \\
& +\int_{0}^{\tau} \int_{\Omega}\left(|\nabla \mathbf{u}|^{2}+\nu|\nabla \times \mathbf{H}|^{2}\right) d x d t+\int_{0}^{\tau} \int_{\Omega} \frac{C}{\theta}\left(\mathbf{S}\left(\theta, \nabla_{x} \mathbf{u}\right): \nabla_{x} \mathbf{u}-\frac{\mathbf{q}\left(\theta, \nabla_{x} \theta\right) \cdot \nabla_{x} \theta}{\theta}\right) d x d t \\
\leq & \int_{\Omega}\left(\frac{1}{2} \rho_{0}\left|\mathbf{u}_{0}-B(0, \cdot)\right|^{2}+\rho_{0} e\left(\rho_{0}, \theta_{0}\right)+\frac{1}{2}\left|\mathbf{H}_{0}\right|^{2}+C(0, \cdot) \rho_{0} s\left(\rho_{0}, \theta_{0}\right)\right) d x \\
& +\int_{0}^{\tau} \int_{\Omega}\left(\left(\rho \partial_{t} B+\rho \mathbf{u} \cdot \nabla_{x} B\right) \cdot(B-\mathbf{u})-P(\rho, \theta) \operatorname{div}_{x} B+\mathbf{S}\left(\theta, \nabla_{x} \mathbf{u}\right): \nabla_{x} B-((\nabla \times \mathbf{H}) \times \mathbf{H}) \cdot B\right) d x d t \\
& -\int_{0}^{\tau} \int_{\Omega}\left(\rho s(\rho, \theta) \partial_{t} C+\rho s(\rho, \theta) \mathbf{u} \cdot \nabla_{x} C+\frac{\mathbf{q}\left(\theta, \nabla_{x} \theta\right)}{\theta} \cdot \nabla_{x} C\right) d x d t .
\end{aligned}
$$

Taking a test function $\varphi=D$ in (35) and $\varphi=\partial_{\rho} H_{C}(A, C)$ in (31), we have

$$
\begin{gathered}
\int_{\Omega} \mathbf{H}(\tau, \cdot) D(\tau, \cdot) d x-\int_{\Omega} \mathbf{H}_{0} D_{0} d x=\int_{0}^{\tau} \int_{\Omega}\left(\mathbf{H} \cdot \partial_{t} D-(\mathbf{H} \times \mathbf{u}+\nu \nabla \times \mathbf{H}) \cdot(\nabla \times D)\right) d x d t,(44) \\
\begin{aligned}
\int_{\Omega} \rho \partial_{\rho} H_{C}(A, C)(\tau, \cdot) d x & -\int_{\Omega} \rho_{0} \partial_{\rho} H_{C(0, \cdot)}(A(0, \cdot), C(0, \cdot)) d x \\
& =\int_{0}^{\tau} \int_{\Omega}\left(\rho \partial_{t}\left(\partial_{\rho} H_{C}(A, C)\right)\right)+\rho \mathbf{u} \cdot \nabla_{x}\left(\partial_{\rho} H_{C}(A, C)\right) d x d t,(45)
\end{aligned}
\end{gathered}
$$

Multiplying (38) by $D$ and integrate over $(0, \tau) \times \Omega$, we find

$$
\int_{\Omega} \frac{1}{2}|D|^{2}(\tau, \cdot) d x-\int_{\Omega} \frac{1}{2}\left|D_{0}\right|^{2} d x=-\int_{0}^{\tau} \int_{\Omega}((D \times B+\nu \nabla \times D) \cdot(\nabla \times D)) d x d t .(46)
$$

So by (43)-(46), we have

$$
\begin{aligned}
& \int_{\Omega}\left(\frac{1}{2} \rho|\mathbf{u}-B|^{2}+\frac{1}{2}|\mathbf{H}-D|^{2}+H_{C}(\rho, \theta)-\partial_{\rho}\left(H_{C}\right)(A, C)(\rho-A)-H_{C}(A, C)\right)(\tau, \cdot) d x \\
& +\int_{0}^{\tau} \int_{\Omega}\left(|\nabla \mathbf{u}|^{2}+\nu\left(|\nabla \times D|^{2}-|\nabla \times D||\nabla \times \mathbf{H}|+|\nabla \times \mathbf{H}|^{2}\right)\right) d x d t \\
& +\int_{0}^{\tau} \int_{\Omega} \frac{C}{\theta}\left(\mathbf{S}\left(\theta, \nabla_{x} \mathbf{u}\right): \nabla_{x} \mathbf{u}-\frac{\mathbf{q}\left(\theta, \nabla_{x} \theta\right) \cdot \nabla_{x} \theta}{\theta}\right) d x d t
\end{aligned}
$$




$$
\begin{aligned}
\leq & \int_{\Omega}\left(\frac{1}{2} \rho_{0}\left|\mathbf{u}_{0}-B(0, \cdot)\right|^{2}+\frac{1}{2}\left|\mathbf{H}_{0}-D_{0}\right|^{2}+\left(H_{C(0, \cdot)}(\rho(0, \cdot), \theta(0, \cdot))\right.\right. \\
& \left.\left.-\partial_{\rho}\left(H_{C(0, \cdot)}\right)(A(0, \cdot), C(0, \cdot))\left(\rho_{0}-A(0, \cdot)\right)-H_{C(0, \cdot)}(A(0, \cdot), C(0, \cdot))\right)\right) d x \\
& +\int_{0}^{\tau} \int_{\Omega}\left(\left(\rho \partial_{t} B+\rho \mathbf{u} \cdot \nabla_{x} B\right) \cdot(B-\mathbf{u})-P(\rho, \theta) \mathbf{d i v}_{x} B+\mathbf{S}\left(\theta, \nabla_{x} \mathbf{u}\right): \nabla_{x} B\right) d x d t \\
& +\int_{0}^{\tau} \int_{\Omega}\left(-\mathbf{H} \cdot \partial_{t} D-((\nabla \times \mathbf{H}) \times \mathbf{H}) \cdot B-(D \times B) \cdot(\nabla \times D)+(\mathbf{H} \times \mathbf{u}) \cdot(\nabla \times D)\right) d x d t \\
& -\int_{0}^{\tau} \int_{\Omega}\left(\rho s(\rho, \theta) \partial_{t} C+\rho s(\rho, \theta) \mathbf{u} \cdot \nabla_{x} C+\frac{\mathbf{q}\left(\theta, \nabla_{x} \theta\right)}{\theta} \cdot \nabla_{x} C\right) d x d t \\
& -\int_{0}^{\tau} \int_{\Omega}\left(\rho \partial_{t}\left(\partial_{\rho}\left(H_{C}\right)(A, C)\right)\right)+\rho \mathbf{u} \cdot \nabla_{x}\left(\partial_{\rho} H_{C}(A, C)\right) d x d t \\
& +\int_{0}^{\tau} \int_{\Omega} \partial_{t}\left(A \partial_{\rho}\left(H_{C}\right)(A, C)-H_{C}(A, C)\right) d x d t .
\end{aligned}
$$

Replacing $\partial_{t} D$ by (38) in (47) to find

$$
\begin{aligned}
& \int_{\Omega}\left(\frac{1}{2} \rho|\mathbf{u}-B|^{2}+\frac{1}{2}|\mathbf{H}-D|^{2}+H_{C}(\rho, \theta)-\partial_{\rho}\left(H_{C}\right)(A, C)(\rho-A)-H_{C}(A, C)\right)(\tau, \cdot) d x \\
& +\int_{0}^{\tau} \int_{\Omega}\left(|\nabla \mathbf{u}|^{2}+\nu|\nabla \times D-\nabla \times \mathbf{H}|^{2}\right) d x d t+\int_{0}^{\tau} \int_{\Omega} \frac{C}{\theta}\left(\mathbf{S}\left(\theta, \nabla_{x} \mathbf{u}\right): \nabla_{x} \mathbf{u}-\frac{\mathbf{q}\left(\theta, \nabla_{x} \theta\right) \cdot \nabla_{x} \theta}{\theta}\right) d x d t \\
\leq & \int_{\Omega}\left(\frac{1}{2} \rho_{0}\left|\mathbf{u}_{0}-B(0, \cdot)\right|^{2}+\frac{1}{2}\left|\mathbf{H}_{0}-D_{0}\right|^{2}+\frac{1}{2} \rho_{0}\left|B_{0}\right|^{2}+\left(H_{C(0, \cdot)}(\rho(0, \cdot), \theta(0, \cdot))\right.\right. \\
& \left.\left.-\partial_{\rho}\left(H_{C(0, \cdot)}\right)(A(0, \cdot), C(0, \cdot))\left(\rho_{0}-A(0, \cdot)\right)-H_{C(0, \cdot)}(A(0, \cdot), C(0, \cdot))\right)\right) d x \\
& \left.+\int_{0}^{\tau} \int_{\Omega}\left(\left(\rho \partial_{t} B+\rho \mathbf{u} \cdot \nabla_{x} B\right) \cdot(B-\mathbf{u})\right)-P(\rho, \theta) \operatorname{div}_{x} B+\mathbf{S}\left(\theta, \nabla_{x} \mathbf{u}\right): \nabla_{x} B\right) d x d t \\
& +\int_{0}^{\tau} \int_{\Omega}(-((\nabla \times \mathbf{H}) \times \mathbf{H}) \cdot B-(D \times B) \cdot(\nabla \times D)+(\mathbf{H} \times \mathbf{u}) \cdot(\nabla \times D)) d x d t \\
& -\int_{0}^{\tau} \int_{\Omega}(\nabla \times(B \times D)) \cdot \mathbf{H} d x d t-\int_{0}^{\tau} \int_{\Omega}\left(\rho s(\rho, \theta) \partial_{t} C+\rho s(\rho, \theta) \mathbf{u} \cdot \nabla_{x} C+\frac{\mathbf{q}\left(\theta, \nabla_{x} \theta\right)}{\theta} \cdot \nabla_{x} C\right) d x d t \\
& -\int_{0}^{\tau} \int_{\Omega}\left(\rho \partial_{t}\left(\partial_{\rho}\left(H_{C}\right)(A, C)\right)\right)+\rho \mathbf{u} \cdot \nabla_{x}\left(\partial_{\rho} H_{C}(A, C)\right) d x d t \\
& +\int_{0}^{\tau} \int_{\Omega} \partial_{t}\left(A \partial_{\rho}\left(H_{C}\right)(A, C)-H_{C}(A, C)\right) d x d t .
\end{aligned}
$$

Note that

$$
\begin{gathered}
\int_{\Omega}((\nabla \times \mathbf{H}) \times \mathbf{H}) \cdot B d x=-\int_{\Omega}\left(\mathbf{H}^{\top} B \mathbf{H}+\frac{1}{2} \nabla\left(|\mathbf{H}|^{2}\right) \cdot B\right) d x, \\
\int_{\Omega}(\nabla \times(B \times \mathbf{H})) \cdot B d x=\int_{\Omega}\left(\mathbf{H}^{\top} B \mathbf{H}+\frac{1}{2} \nabla\left(|\mathbf{H}|^{2}\right) \cdot B\right) d x .
\end{gathered}
$$


So direct calculation shows that

$$
\begin{aligned}
& \int_{0}^{\tau} \int_{\Omega}(-((\nabla \times \mathbf{H}) \times \mathbf{H}) \cdot B-(D \times B) \cdot(\nabla \times D)+(\mathbf{H} \times \mathbf{u}) \cdot(\nabla \times D)) d x d t \\
& -\int_{0}^{\tau} \int_{\Omega} \nabla \times(B \times D) \cdot \mathbf{H} d x d t \\
= & \int_{0}^{\tau} \int_{\Omega}(-((\nabla \times \mathbf{H}) \times \mathbf{H}) \cdot B-\nabla \times(B \times D) \cdot \mathbf{H}) d x d t \\
& +\int_{0}^{\tau} \int_{\Omega}(-(D \times B) \cdot(\nabla \times D)+(\mathbf{H} \times \mathbf{u}) \cdot(\nabla \times D)) d x d t \\
= & \int_{0}^{\tau} \int_{\Omega}\left((\mathbf{H}-D)^{\top} \nabla B(\mathbf{H}-D)+\frac{1}{2} \nabla\left(|\mathbf{H}-D|^{2}\right) \cdot B\right) d x d t \\
& +\int_{0}^{\tau} \int_{\Omega}(D-\mathbf{H})^{\top} \nabla(\mathbf{u}-B) D+\frac{1}{2} \nabla(D(D-\mathbf{H}))(\mathbf{u}-B) d x d t \\
& -\int_{0}^{\tau} \int_{\Omega}\left(D^{\top} \nabla(\mathbf{u}-B) D+\frac{1}{2} \nabla\left(|D|^{2}\right)(\mathbf{u}-B)\right) d x d t .
\end{aligned}
$$

Note that

$$
\begin{aligned}
\partial_{y}\left(\partial_{\rho} H_{C}(A, C)\right)= & -s(A, C) \partial_{y} C-A \partial_{\rho} s(A, C) \partial_{y} C+\partial_{\rho, \rho}^{2} H_{C}(A, C) \partial_{y} \rho \\
& +\partial_{\rho, \theta}^{2} H_{C}(A, C) \partial_{y} C, \quad \text { for } y=t, x .
\end{aligned}
$$

Thus it follows from (48) and (50) that

$$
\begin{aligned}
& \int_{\Omega}\left(\frac{1}{2} \rho|\mathbf{u}-B|^{2}+\frac{1}{2}|\mathbf{H}-D|^{2}+H_{C}(\rho, \theta)-\partial_{\rho}\left(H_{C}\right)(A, C)(\rho-A)-H_{C}(A, C)\right)(\tau, \cdot) d x \\
& +\int_{0}^{\tau} \int_{\Omega}\left(|\nabla \mathbf{u}|^{2}+\nu|\nabla \times D-\nabla \times \mathbf{H}|^{2}\right) d x d t+\int_{0}^{\tau} \int_{\Omega} \frac{C}{\theta}\left(\mathbf{S}\left(\theta, \nabla_{x} \mathbf{u}\right): \nabla_{x} \mathbf{u}-\frac{\mathbf{q}\left(\theta, \nabla_{x} \theta\right) \cdot \nabla_{x} \theta}{\theta}\right) d x d t \\
\leq & \int_{\Omega}\left(\frac{1}{2} \rho_{0}\left|\mathbf{u}_{0}-B(0, \cdot)\right|^{2}+\frac{1}{2}\left|\mathbf{H}_{0}-D_{0}\right|^{2}+\left(H_{C(0, \cdot)}(\rho(0, \cdot), \theta(0, \cdot))\right.\right. \\
& \left.\left.-\partial_{\rho}\left(H_{C(0, \cdot)}\right)(A(0, \cdot), C(0, \cdot))\left(\rho_{0}-A(0, \cdot)\right)-H_{C(0, \cdot)}(A(0, \cdot), C(0, \cdot))\right)\right) d x \\
& +\int_{0}^{\tau} \int_{\Omega}\left(\left(\rho \partial_{t} B+\rho \mathbf{u} \cdot \nabla_{x} B\right) \cdot(B-\mathbf{u})-P(\rho, \theta) \mathbf{d i v}_{x} B+\mathbf{S}\left(\theta, \nabla_{x} \mathbf{u}\right): \nabla_{x} B\right) d x d t \\
& +\int_{0}^{\tau} \int_{\Omega}\left((\mathbf{H}-D)^{\top} \nabla B(\mathbf{H}-D)+\frac{1}{2} \nabla\left(|\mathbf{H}-D|^{2}\right) \cdot B\right) d x d t \\
& +\int_{0}^{\tau} \int_{\Omega}(D-\mathbf{H})^{\top} \nabla(\mathbf{u}-B) D+\frac{1}{2} \nabla(D(D-\mathbf{H}))(\mathbf{u}-B) d x d t \\
& -\int_{0}^{\tau} \int_{\Omega}\left(D^{\top} \nabla(\mathbf{u}-B) D+\frac{1}{2} \nabla\left(|D|^{2}\right)(\mathbf{u}-B)\right) d x d t \\
& -\int_{0}^{\tau} \int_{\Omega}\left(\rho(s(\rho, \theta)-s(A, C)) \partial_{t} C+\rho(s(\rho, \theta)-s(A, C)) \mathbf{u} \cdot \nabla_{x} C+\frac{\mathbf{q}\left(\theta, \nabla_{x} \theta\right)}{\theta} \cdot \nabla_{x} C\right) d x d t \\
& +\int_{0}^{\tau} \int_{\Omega} \rho\left(A \partial_{\rho} s(A, C) \partial_{t} C+r \partial_{\rho} s(A, C) \mathbf{u} \cdot \nabla_{x} C\right) d x d t
\end{aligned}
$$




$$
\begin{aligned}
& -\int_{0}^{\tau} \int_{\Omega} \rho\left(\partial_{\rho, \rho}^{2}\left(H_{C}\right)(A, C) \partial_{t} A+\partial_{\rho, \theta}^{2}\left(H_{C}\right)(A, C) \partial_{t} C\right) d x d t \\
& -\int_{0}^{\tau} \int_{\Omega} \rho \mathbf{u}\left(\partial_{\rho, \rho}^{2}\left(H_{C}\right)(A, C) \nabla_{x} A+\partial_{\rho, \theta}^{2}\left(H_{C}\right)(A, C) \nabla_{x} C\right) d x d t \\
& +\int_{0}^{\tau} \int_{\Omega} \partial_{t}\left(A \partial_{\rho}\left(H_{C}\right)(A, C)-H_{C}(A, C)\right) d x d t .
\end{aligned}
$$

Following [1,10,20, introducing the quantity as

$$
\Gamma(\rho, \theta \mid C, C)=H_{C}(\rho, \theta)-\partial_{\rho} H_{C}(A, C)(\rho-A)-H_{C}(A, C),
$$

where

$$
H_{C}(\rho, \theta)=\rho e(\rho, \theta)-C \rho s(\rho, \theta) .
$$

Note that

$$
\begin{aligned}
& \partial_{\rho, \rho}^{2} H_{C}(A, C)=\frac{1}{A} \partial_{\rho} P(A, C), \quad A \partial_{\rho} s(A, C)=-\frac{1}{C} \partial_{\theta} P(A, C), \\
& \partial_{\rho, \theta}^{2} H_{C}(A, C)=\partial_{\rho}\left(\rho(\theta-C) \partial_{\theta} s\right)(A, C)=(\theta-C) \partial_{\rho}\left(\rho \partial_{\theta} s(\rho, \theta)\right)(A, C)=0, \\
& A \partial_{\rho}\left(H_{C}\right)(A, C)-H_{C}(A, C)=P(A, C) .
\end{aligned}
$$

Therefore, we can obtain a kind of relative entropy inequality by simplifying (51) as

$$
\begin{aligned}
& \int_{\Omega}\left(\frac{1}{2} \rho|\mathbf{u}-B|^{2}+\frac{1}{2}|\mathbf{H}-D|^{2}+\Gamma(\rho, \theta \mid A, C)\right)(\tau, \cdot) d x+\nu \int_{0}^{\tau} \int_{\Omega}|\nabla \times D-\nabla \times \mathbf{H}|^{2} d x d t \\
& +\int_{0}^{\tau} \int_{\Omega} \frac{C}{\theta}\left(\mathbf{S}\left(\theta, \nabla_{x} \mathbf{u}\right): \nabla_{x} \mathbf{u}-\frac{\mathbf{q}\left(\theta, \nabla_{x} \theta\right) \cdot \nabla_{x} \theta}{\theta}\right) d x d t \\
\leq & \int_{\Omega}\left(\frac{1}{2} \rho_{0}\left|\mathbf{u}_{0}-B(0, \cdot)\right|^{2}+\frac{1}{2}\left|\mathbf{H}_{0}-D_{0}(0, \cdot)\right|^{2}+\Gamma\left(\rho_{0}, \theta_{0} \mid A(0, \cdot), C(0, \cdot)\right)\right) \\
& -\int_{0}^{\tau} \int_{\Omega} \rho(\mathbf{u}-B) \cdot \nabla_{x} B \cdot(B-\mathbf{u}) d x d t+\int_{0}^{\tau} \int_{\Omega} \rho(s(\rho, \theta)-s(A, C))(B-\mathbf{u}) \cdot \nabla_{x} C d x d t \\
& +\int_{0}^{\tau} \int_{\Omega}\left(\left(\rho \partial_{t} B+\rho \mathbf{u} \cdot \nabla_{x} B\right) \cdot(B-\mathbf{u})-P(\rho, \theta) \mathbf{d i v}_{x} B+\mathbf{S}\left(\theta, \nabla_{x} \mathbf{u}\right): \nabla_{x} B\right) d x d t \\
& +\int_{0}^{\tau} \int_{\Omega}\left((\mathbf{H}-D)^{\top} \nabla B(\mathbf{H}-D)+\frac{1}{2} \nabla\left(|\mathbf{H}-D|^{2}\right) \cdot B\right) d x d t \\
& +\int_{0}^{\tau} \int_{\Omega}(D-\mathbf{H})^{\top} \nabla(\mathbf{u}-B) D+\frac{1}{2} \nabla(D(D-\mathbf{H}))(\mathbf{u}-B) d x d t \\
& -\int_{0}^{\tau} \int_{\Omega}\left(D^{\top} \nabla(\mathbf{u}-B) D+\frac{1}{2} \nabla\left(|D|^{2}\right)(\mathbf{u}-B)\right) d x d t \\
& -\int_{0}^{\tau} \int_{\Omega}\left(\rho(s(\rho, \theta)-s(A, C)) \partial_{t} C+\rho(s(\rho, \theta)-s(A, C)) \mathbf{u} \cdot \nabla_{x} C+\frac{\mathbf{q}\left(\theta, \nabla_{x} \theta\right)}{\theta} \cdot \nabla_{x} C\right) d x d t \\
& +\int_{0}^{\tau} \int_{\Omega}\left(\left(1-\frac{\rho}{A}\right) \partial_{t} P(A, C)-\frac{\rho}{A} \mathbf{u} \cdot \nabla_{x} P(A, C)\right) d x d t .
\end{aligned}
$$


Now we state the weak-strong uniqueness property to the full magnetohydrodynamic system (11)-(4) on a bounded Lipschitz domains with Dirichlet boundary conditions.

Theorem 1 Let $\Omega \subset \boldsymbol{R}^{3}$ be a bounded Lipschitz domain and $(\rho, \boldsymbol{u}, \theta, \boldsymbol{H})$ be a weak solution of the full magnetohydrodynamic system (11)-(4) in $(0, T) \times \Omega$ and $\left(\rho^{\prime}, \boldsymbol{u}^{\prime}, \theta^{\prime}, \boldsymbol{H}^{\prime}\right)$ be a strong solution emanating from the same initial data (37). Assume that the thermodynamic functions $P, e, s$ satisfy hypotheses (19)-(28), and that the transport coefficients $\mu, \eta$ and $\kappa$ satisfy (29)-(30). Then

$$
\rho \equiv \rho^{\prime}, \quad \boldsymbol{u}=\boldsymbol{u}^{\prime}, \quad \theta=\theta^{\prime}, \quad \boldsymbol{H}=\boldsymbol{H}^{\prime} .
$$

\section{Proof of Theorem 1}

In this section, we apply the relative entropy inequality to finish the proof of Theorem 1. Assume that $\left(\rho^{\prime}, \mathbf{u}^{\prime}, \theta^{\prime}, \mathbf{H}^{\prime}\right)$ is a classical (strong) solution to the full magnetohydrodynamic system in $(0, T) \times \Omega$, it satisfies that

$$
\rho^{\prime}(0, \cdot)=\rho_{0}, \quad \mathbf{u}^{\prime}(0, \cdot)=\mathbf{u}_{0}, \quad \theta^{\prime}(0, \cdot)=\theta_{0}, \quad \mathbf{H}^{\prime}(0, \cdot)=\mathbf{H}_{0} .
$$

Following [7, 10, we introduce essential and residual component of each quantity appearing in (52). Thermodynamic stability hypothesis (24) implies that $\rho \mapsto H_{\theta^{\prime}}\left(\rho, \theta^{\prime}\right)$ is strictly convex, while $\theta \mapsto H_{\theta^{\prime}}\left(\rho, \theta^{\prime}\right)$ attains its global minimum at $\theta=\theta^{\prime}$. Thus it has

$$
\Gamma\left(\rho, \theta \mid \rho^{\prime}, \theta^{\prime}\right) \geq c \begin{cases}\left|\rho-\rho^{\prime}\right|^{2}+\left|\theta-\theta^{\prime}\right|^{2} \quad \text { if } & (\rho, \theta) \in\left[\rho_{0}^{\prime}, \rho_{1}^{\prime}\right] \times\left[\theta_{0}^{\prime}, \theta_{1}^{\prime}\right] \\ 1+|\rho s(\rho, \theta)|+\rho e(\rho, \theta) & \text { otherwise, }\end{cases}
$$

where $\left[\rho^{\prime}, \theta^{\prime}\right] \in\left[\rho_{0}^{\prime}, \rho_{1}^{\prime}\right] \times\left[\theta_{0}^{\prime}, \theta_{1}^{\prime}\right]$, the constant $c$ depends on positive constants $\rho_{0}^{\prime}, \rho_{1}^{\prime}, \theta_{0}^{\prime}, \theta_{1}^{\prime}$ and the structural properties of the thermodynamic function $e, s$. More precisely, the restriction of positive constants $\rho_{0}^{\prime}, \rho_{1}^{\prime}, \theta_{0}^{\prime}, \theta_{1}^{\prime}$ can be found in $[10]$.

Thus we can write each measurable function $h=h_{e s s}+h_{r e s}$, where

$$
h_{\text {ess }}=\left\{\begin{array}{l}
h(t, x) \text { if }(\rho, \theta) \in\left[\rho_{0}^{\prime}, \rho_{1}^{\prime}\right] \times\left[\theta_{0}^{\prime}, \theta_{1}^{\prime}\right] \\
0 \text { otherwise. }
\end{array}\right.
$$

Taking $(A, B, C, D)=\left(\rho^{\prime}, \mathbf{u}^{\prime}, \theta^{\prime}, \mathbf{H}^{\prime}\right)$ in (53). By the fact that the initial data coincide, we have

$$
\begin{aligned}
& \int_{\Omega}\left(\frac{1}{2} \rho\left|\mathbf{u}-\mathbf{u}^{\prime}\right|^{2}+\frac{1}{2}\left|\mathbf{H}-\mathbf{H}^{\prime}\right|^{2}+\Gamma\left(\rho, \theta \mid \rho^{\prime}, \theta\right)\right)(\tau, \cdot) d x+\nu \int_{0}^{\tau} \int_{\Omega}\left|\nabla \times \mathbf{H}^{\prime}-\nabla \times \mathbf{H}\right|^{2} d x d t \\
& +\int_{0}^{\tau} \int_{\Omega} \frac{\theta^{\prime}}{\theta}\left(\mathbf{S}\left(\theta, \nabla_{x} \mathbf{u}\right): \nabla_{x} \mathbf{u}-\frac{\mathbf{q}\left(\theta, \nabla_{x} \theta\right) \cdot \nabla_{x} \theta}{\theta}\right) d x d t \\
\leq & \int_{\Omega}\left(\frac{1}{2} \rho_{0}\left|\mathbf{u}_{0}-\mathbf{u}^{\prime}(0, \cdot)\right|^{2}+\frac{1}{2}\left|\mathbf{H}_{0}-\mathbf{H}_{0}^{\prime}(0, \cdot)\right|^{2}+\Gamma\left(\rho_{0}, \theta_{0} \mid \rho^{\prime}(0, \cdot), \theta^{\prime}(0, \cdot)\right)\right) \\
& +\int_{0}^{\tau} \int_{\Omega} \rho\left|\mathbf{u}-\mathbf{u}^{\prime}\right|^{2}\left|\nabla_{x} \mathbf{u}^{\prime}\right| d x d t+\int_{0}^{\tau} \int_{\Omega} \rho(s(\rho, \theta)-s(\rho, \theta))\left(\mathbf{u}^{\prime}-\mathbf{u}\right) \cdot \nabla_{x} \theta^{\prime} d x d t
\end{aligned}
$$




$$
\begin{aligned}
& +\int_{0}^{\tau} \int_{\Omega}\left(\left(\rho \partial_{t} \mathbf{u}^{\prime}+\rho \mathbf{u} \cdot \nabla_{x} \mathbf{u}^{\prime}\right) \cdot\left(\mathbf{u}^{\prime}-\mathbf{u}\right)-P(\rho, \theta) \mathbf{d i v} \mathbf{v}_{x} \mathbf{u}^{\prime}+\mathbf{S}\left(\theta, \nabla_{x} \mathbf{u}\right): \nabla_{x} \mathbf{u}^{\prime}\right) d x d t \\
& +\int_{0}^{\tau} \int_{\Omega}\left(\left(\mathbf{H}-\mathbf{H}^{\prime}\right)^{\top} \nabla \mathbf{u}^{\prime}\left(\mathbf{H}-\mathbf{H}^{\prime}\right)+\frac{1}{2} \nabla\left(\left|\mathbf{H}-\mathbf{H}^{\prime}\right|^{2}\right) \cdot \mathbf{u}^{\prime}\right) d x \\
& +\int_{0}^{\tau} \int_{\Omega}\left(\mathbf{H}^{\prime}-\mathbf{H}\right)^{\top} \nabla\left(\mathbf{u}-\mathbf{u}^{\prime}\right) \mathbf{H}^{\prime}+\frac{1}{2} \nabla\left(\mathbf{H}^{\prime}\left(\mathbf{H}^{\prime}-\mathbf{H}\right)\right)\left(\mathbf{u}-\mathbf{u}^{\prime}\right) d x d t \\
& -\int_{0}^{\tau} \int_{\Omega}\left(\mathbf{H}^{\prime \top} \nabla\left(\mathbf{u}-\mathbf{u}^{\prime}\right) \mathbf{H}^{\prime}+\frac{1}{2} \nabla\left(\left|\mathbf{H}^{\prime}\right|^{2}\right)\left(\mathbf{u}-\mathbf{u}^{\prime}\right)\right) d x d t \\
& -\int_{0}^{\tau} \int_{\Omega}\left(\rho\left(s(\rho, \theta)-s\left(\rho^{\prime}, \theta^{\prime}\right)\right) \partial_{t} \theta^{\prime}+\rho\left(s(\rho, \theta)-s\left(\rho^{\prime}, \theta^{\prime}\right)\right) \mathbf{u}^{\prime} \cdot \nabla_{x} \theta^{\prime}+\frac{\mathbf{q}\left(\theta, \nabla_{x} \theta\right)}{\theta} \cdot \nabla_{x} \theta^{\prime}\right) d x d t \\
& +\int_{0}^{\tau} \int_{\Omega}\left(\left(1-\frac{\rho}{\rho^{\prime}}\right) \partial_{t} P\left(\rho^{\prime}, \theta^{\prime}\right)-\frac{\rho}{\rho^{\prime}} \mathbf{u} \cdot \nabla_{x} P\left(\rho^{\prime}, \theta^{\prime}\right)\right) d x d t .
\end{aligned}
$$

In what follows, we estimate the right-hand side of (55). It is easy to see that

$$
\int_{\Omega} \rho\left|\mathbf{u}-\mathbf{u}^{\prime}\right|^{2}\left|\nabla_{x} \mathbf{u}^{\prime}\right| d x \leq\left\|\nabla_{x} \mathbf{u}^{\prime}\right\|_{\mathbf{L}^{\infty}\left(\Omega ; \mathbf{R}^{3}\right)} \int_{\Omega} \rho\left|\mathbf{u}-\mathbf{u}^{\prime}\right|^{2} d x
$$

By virtue of (54), using interpolation inequality, for any $\epsilon>0$, we derive

$$
\begin{aligned}
& \int_{\Omega} \rho(s(\rho, \theta)-s(\rho, \theta))\left(\mathbf{u}^{\prime}-\mathbf{u}\right) \cdot \nabla_{x} \theta^{\prime} d x \\
\leq & 2 \rho_{1}^{\prime}\left\|\nabla_{x} \theta^{\prime}\right\|_{\mathbf{L}^{\infty}\left(\Omega ; \mathbf{R}^{3}\right)}\left(\epsilon\left\|\mathbf{u}^{\prime}-\mathbf{u}\right\|_{\mathbf{L}^{2}\left(\Omega ; \mathbf{R}^{3}\right)}^{2}+c(\epsilon) \int_{\Omega} \Gamma\left(\rho, \theta \mid \rho^{\prime}, \theta^{\prime}\right) d x\right) \\
& +\left\|\nabla_{x} \theta^{\prime}\right\|_{\left.\mathbf{L}_{(\Omega ;}^{\infty} ; \mathbf{R}^{3}\right)}\left(\epsilon\left\|\mathbf{u}^{\prime}-\mathbf{u}\right\|_{\left.\mathbf{L}_{(\Omega ;}^{6} ; \mathbf{R}^{3}\right)}^{2}+c(\epsilon)\left\|\left[\rho\left(s(\rho, \theta)-s\left(\rho^{\prime}, \theta^{\prime}\right)\right)\right]_{\text {res }}\right\|_{\mathbf{L}^{\frac{6}{5}}(\Omega)}^{2}\right) .
\end{aligned}
$$

It follows from (26)-(27) that

$$
\left|\left[\rho\left(s(\rho, \theta)-s\left(\rho^{\prime}, \theta^{\prime}\right)\right)\right]_{r e s}\right| \leq c\left(\rho+\rho[\log \theta]^{+}+\rho|\log \rho|+\theta^{3}\right) .
$$

Using (22), (25)-(26),

$$
\rho e(\rho, \theta) \geq c\left(\rho^{\frac{5}{3}}+\theta^{4}\right)
$$

and (33)-(36) imply

$$
t \mapsto \int_{\Omega} \Gamma\left(\rho, \theta \mid \rho^{\prime}, \theta^{\prime}\right) d x \in \mathbf{L}^{\infty}(0, T) .
$$

By (54), (58)-(60) and Hölder inequality,

$$
\left\|\left[\rho\left(s(\rho, \theta)-s\left(\rho^{\prime}, \theta^{\prime}\right)\right)\right]_{r e s}\right\|_{\mathbf{L}^{\frac{6}{5}(\Omega)}}^{2} \leq c\left(\int_{\Omega} \Gamma\left(\rho, \theta \mid \rho^{\prime}, \theta,\right)\right)^{\frac{5}{3}} .
$$

So by (57), for any $\epsilon>0$, we obtain

$$
\int_{\Omega} \rho(s(\rho, \theta)-s(\rho, \theta))\left(\mathbf{u}^{\prime}-\mathbf{u}\right) \cdot \nabla_{x} \theta^{\prime} d x \leq \epsilon\left\|\mathbf{u}^{\prime}-\mathbf{u}\right\|_{\mathbf{W}_{0}^{1,2}\left(\Omega ; \mathbf{R}^{3}\right)}^{2}+c^{\prime}(\epsilon, \cdot) \int_{\Omega} \Gamma\left(\rho, \theta \mid \rho^{\prime}, \theta^{\prime}\right) d x,(61)
$$


where $c^{\prime}(\epsilon, \cdot)$ is a generic constant depending on $\epsilon, \rho^{\prime}, \mathbf{u}^{\prime}$ and $\theta^{\prime}$ through the norms induced by (36)-(37), while $c^{\prime}(\cdot)$ is independent of $\epsilon$ but depends on $\rho^{\prime}$, $\mathbf{u}^{\prime}, \theta^{\prime}, \rho_{0}^{\prime}$ and $\theta_{0}$, through the norms induced by (36)-(37).

Similar with estimating (61), we get

$$
\begin{aligned}
& \left.\int_{\Omega} \frac{1}{\rho^{\prime}}\left(\rho-\rho^{\prime}\right)\left(\mathbf{u}^{\prime}-\mathbf{u}^{\prime}\right) \cdot\left(\operatorname{div}_{x} \mathbf{S}\left(\theta^{\prime}, \nabla_{x} \mathbf{u}\right)-\nabla_{x} P\left(\rho^{\prime}, \theta^{\prime}\right)+\left(\nabla \times \mathbf{H}^{\prime}\right) \times \mathbf{H}^{\prime}\right)\right) d x \\
= & \int_{\Omega}\left[\rho^{\prime-1}\left(\rho-\rho^{\prime}\right)\left(\mathbf{u}^{\prime}-\mathbf{u}^{\prime}\right) \cdot\left[\operatorname{div}_{x} \mathbf{S}\left(\theta^{\prime}, \nabla_{x} \mathbf{u}\right)-\nabla_{x} P\left(\rho^{\prime}, \theta^{\prime}\right)+\left(\nabla \times \mathbf{H}^{\prime}\right) \times \mathbf{H}^{\prime}\right)\right]_{e s s} d x \\
& +\int_{\Omega}\left[\rho^{\prime-1}\left(\rho-\rho^{\prime}\right)\left(\mathbf{u}^{\prime}-\mathbf{u}^{\prime}\right) \cdot\left[\operatorname{div}_{x} \mathbf{S}\left(\theta^{\prime}, \nabla_{x} \mathbf{u}\right)-\nabla_{x} P\left(\rho^{\prime}, \theta^{\prime}\right)+\left(\nabla \times \mathbf{H}^{\prime}\right) \times \mathbf{H}^{\prime}\right)\right]_{e s s} d x \\
\leq & c^{\prime}(\epsilon, \cdot)\left\|\left[\rho-\rho^{\prime}\right]_{e s s}\right\|_{\mathbf{L}^{2}(\Omega)}^{2}+\epsilon\left\|\mathbf{u}^{\prime}-\mathbf{u}\right\|_{\mathbf{L}^{2}\left(\Omega ; \mathbf{R}^{3}\right)}^{2} \\
& +c^{\prime}(\epsilon, \cdot)\left(\left\|[\rho]_{e s s}\right\|_{\mathbf{L}^{\frac{6}{5}}(\Omega)}^{2}+\left\|[1]_{r e s}\right\|_{\mathbf{L}^{\frac{6}{5}}(\Omega)}^{2}\right)+\epsilon\left\|\mathbf{u}^{\prime}-\mathbf{u}\right\|_{\mathbf{L}^{6}\left(\Omega ; \mathbf{R}^{3}\right)^{\prime}}^{2}
\end{aligned}
$$

So using integrating by parts, then virtue of (54), (60), (62) and $\mathbf{W}^{1,2}(\Omega) \hookrightarrow$ $\mathbf{L}^{6}(\Omega)$, we derive

$$
\begin{aligned}
& \left|\int_{\Omega} \rho\left(\partial_{t} \mathbf{u}^{\prime}+\mathbf{u}^{\prime} \cdot \nabla_{x} \mathbf{u}^{\prime}\right) \cdot\left(\mathbf{u}^{\prime}-\mathbf{u}\right) d x\right| \\
= & \left|\int_{\Omega} \frac{\rho}{\rho^{\prime}}\left(\mathbf{u}^{\prime}-\mathbf{u}\right) \cdot\left(\operatorname{div}_{x} \mathbf{S}\left(\theta^{\prime}, \nabla_{x} \mathbf{u}^{\prime}\right)-\nabla_{x} P\left(\rho^{\prime}, \theta^{\prime}\right)+\left(\nabla \times \mathbf{H}^{\prime}\right) \times \mathbf{H}^{\prime}\right) d x\right| \\
\leq & \int_{\Omega}\left|\frac{\rho-\rho^{\prime}}{\rho^{\prime}}\left(\mathbf{u}^{\prime}-\mathbf{u}\right) \cdot\left(\operatorname{div}_{x} \mathbf{S}\left(\theta^{\prime}, \nabla_{x} \mathbf{u}^{\prime}\right)-\nabla_{x} P\left(\rho^{\prime}, \theta^{\prime}\right)+\left(\nabla \times \mathbf{H}^{\prime}\right) \times \mathbf{H}^{\prime}\right)\right| d x \\
& +\left|\int_{\Omega}\left(\mathbf{u}^{\prime}-\mathbf{u}\right) \cdot\left(\operatorname{div}_{x} \mathbf{S}\left(\theta^{\prime}, \nabla_{x} \mathbf{u}^{\prime}\right)-\nabla_{x} P\left(\rho^{\prime}, \theta^{\prime}\right)+\left(\nabla \times \mathbf{H}^{\prime}\right) \times \mathbf{H}^{\prime}\right) d x\right| \\
\leq & c^{\prime}(\epsilon, \cdot)\left\|\left[\rho-\rho^{\prime}\right]_{e s s}\right\|_{\mathbf{L}^{2}(\Omega)}^{2}+\epsilon\left\|\mathbf{u}^{\prime}-\mathbf{u}\right\|_{\mathbf{L}^{2}\left(\Omega ; \mathbf{R}^{3}\right)}^{2}+c^{\prime}(\epsilon, \cdot)\left(\left\|[\rho]_{e s s}\right\|_{\mathbf{L}^{\frac{6}{5}}(\Omega)}^{2}+\left\|[1]_{r e s}\right\|_{\mathbf{L}^{\frac{6}{5}}(\Omega)}^{2}\right) \\
& +\epsilon\left\|\mathbf{u}^{\prime}-\mathbf{u}\right\|_{\mathbf{L}^{6}\left(\Omega ; \mathbf{R}^{3}\right)} \\
& +\left|\int_{\Omega}\left(\mathbf{S}\left(\theta^{\prime}, \nabla_{x} \mathbf{u}^{\prime}\right): \nabla_{x}\left(\mathbf{u}^{\prime}-\mathbf{u}\right)+P\left(\rho^{\prime}, \theta^{\prime}\right) \operatorname{div}_{x}\left(\mathbf{u}^{\prime}-\mathbf{u}\right)+\left(\left(\nabla \times \mathbf{H}^{\prime}\right) \times \mathbf{H}^{\prime}\right) \cdot\left(\mathbf{u}^{\prime}-\mathbf{u}\right)\right) d x\right| \\
\leq & \left|\int_{\Omega}\left(\mathbf{S}\left(\theta^{\prime}, \nabla_{x} \mathbf{u}^{\prime}\right): \nabla_{x}\left(\mathbf{u}^{\prime}-\mathbf{u}\right)+P\left(\rho^{\prime}, \theta^{\prime}\right) \mathbf{d i v}_{x}\left(\mathbf{u}^{\prime}-\mathbf{u}\right)+\left(\left(\nabla \times \mathbf{H}^{\prime}\right) \times \mathbf{H}^{\prime}\right) \cdot\left(\mathbf{u}^{\prime}-\mathbf{u}\right)\right) d x\right| \\
& +\left(\epsilon\left\|\mathbf{u}-\mathbf{u}^{\prime}\right\|_{\mathbf{W}_{0}^{1,2}\left(\Omega ; \mathbf{R}^{3}\right)}+c(\epsilon) \int_{\Omega} \Gamma\left(\rho, \theta \mid \rho^{\prime}, \theta^{\prime}\right) d x\right) .
\end{aligned}
$$

By Hölder inequality and (13)-(14), we derive

$$
\begin{aligned}
& \int_{0}^{\tau} \int_{\Omega}\left(\left(\mathbf{H}-\mathbf{H}^{\prime}\right)^{\top} \nabla \mathbf{u}^{\prime}\left(\mathbf{H}-\mathbf{H}^{\prime}\right) d x d t \leq \int_{0}^{\tau}\left(\left\|\nabla \mathbf{u}^{\prime}\right\|_{\mathbf{L}^{\infty}\left(\Omega ; \mathbf{R}^{3}\right)} \int_{\Omega}\left|\mathbf{H}-\mathbf{H}^{\prime}\right|^{2} d x\right) d t,\right. \\
& \frac{1}{2} \int_{0}^{\tau} \int_{\Omega} \nabla\left(\left|\mathbf{H}-\mathbf{H}^{\prime}\right|^{2}\right) \cdot \mathbf{u}^{\prime} d x d x d t \leq \frac{1}{2} \int_{0}^{\tau}\left(\left\|\nabla \mathbf{u}^{\prime}\right\|_{\mathbf{L}^{\infty}\left(\Omega ; \mathbf{R}^{3}\right)} \int_{\Omega}\left|\mathbf{H}-\mathbf{H}^{\prime}\right|^{2} d x\right) d t,
\end{aligned}
$$




$$
\begin{aligned}
\int_{0}^{\tau} \int_{\Omega}\left(\mathbf{H}^{\prime}-\mathbf{H}\right)^{\top} \nabla\left(\mathbf{u}-\mathbf{u}^{\prime}\right) \mathbf{H}^{\prime} d x d t \leq & \int_{0}^{\tau} c_{\epsilon}\left\|\mathbf{H}^{\prime}\right\|_{\mathbf{L}^{\infty}\left(\Omega ; \mathbf{R}^{3}\right)} \int_{\Omega}\left|\mathbf{H}^{\prime}-\mathbf{H}\right|^{2} d x d t \\
& +\int_{0}^{\tau} \epsilon\left\|\mathbf{H}^{\prime}\right\|_{\left.\mathbf{L}_{(\Omega ;}^{\infty} ; \mathbf{R}^{3}\right)}\left\|\mathbf{u}-\mathbf{u}^{\prime}\right\|_{\mathbf{W}^{1,2}\left(\Omega ; \mathbf{R}^{3}\right)}^{2} d t
\end{aligned}
$$

$\frac{1}{2} \int_{0}^{\tau} \int_{\Omega} \nabla\left(\mathbf{H}^{\prime}\left(\mathbf{H}^{\prime}-\mathbf{H}\right)\right)\left(\mathbf{u}-\mathbf{u}^{\prime}\right) d x d t \leq \frac{1}{4} \int_{0}^{\tau} c_{\epsilon} \int_{\Omega}\left|\mathbf{H}^{\prime}-\mathbf{H}\right|^{2} d x d t$

$$
+\frac{1}{4} \int_{0}^{\tau} \epsilon\left\|\mathbf{H}^{\prime}\right\|_{\mathbf{L}^{\infty}\left(\Omega ; \mathbf{R}^{3}\right)}^{2}\left\|\mathbf{u}-\mathbf{u}^{\prime}\right\|_{\mathbf{W}^{1,2}\left(\Omega ; \mathbf{R}^{3}\right)}^{2} d t,(67)
$$

Thus combing with (64)-67) and (49), we have

$$
\begin{aligned}
& \int_{0}^{\tau} \int_{\Omega}\left(\left(\mathbf{H}-\mathbf{H}^{\prime}\right)^{\top} \nabla \mathbf{u}^{\prime}\left(\mathbf{H}-\mathbf{H}^{\prime}\right)+\frac{1}{2} \nabla\left(\left|\mathbf{H}-\mathbf{H}^{\prime}\right|^{2}\right) \cdot \mathbf{u}^{\prime}\right) d x \\
& +\int_{0}^{\tau} \int_{\Omega}\left(\mathbf{H}^{\prime}-\mathbf{H}\right)^{\top} \nabla\left(\mathbf{u}-\mathbf{u}^{\prime}\right) \mathbf{H}^{\prime}+\frac{1}{2} \nabla\left(\mathbf{H}^{\prime}\left(\mathbf{H}^{\prime}-\mathbf{H}\right)\right)\left(\mathbf{u}-\mathbf{u}^{\prime}\right) d x d t \\
& -\int_{0}^{\tau} \int_{\Omega}\left(\mathbf{H}^{\prime \top} \nabla\left(\mathbf{u}-\mathbf{u}^{\prime}\right) \mathbf{H}^{\prime}+\frac{1}{2} \nabla\left(\left|\mathbf{H}^{\prime}\right|^{2}\right)\left(\mathbf{u}-\mathbf{u}^{\prime}\right)\right) d x d t \\
\leq & \int_{0}^{\tau}\left(\epsilon c^{\prime \prime}\left\|\mathbf{u}-\mathbf{u}^{\prime}\right\|^{2} \mathbf{W}^{1,2}\left(\Omega ; \mathbf{R}^{3}\right)\right. \\
& \left.+c_{\epsilon} \int_{\Omega}\left|\mathbf{H}^{\prime}-\mathbf{H}\right|^{2} d x\right) d t \\
& +\int_{0}^{\tau} \int_{\Omega}\left(\left(\nabla \times \mathbf{H}^{\prime}\right) \times \mathbf{H}^{\prime}\right)\left(\mathbf{u}-\mathbf{u}^{\prime}\right) d x d t,
\end{aligned}
$$

where $c^{\prime \prime}=c^{\prime \prime}\left(\left\|\mathbf{H}^{\prime}\right\|_{\mathbf{L}^{\infty}\left(\Omega ; \mathbf{R}^{3}\right)}\right)$ and $c_{\epsilon}=c_{\epsilon}\left(\left\|\nabla \mathbf{u}^{\prime}\right\|_{\mathbf{L}^{\infty}\left(\Omega ; \mathbf{R}^{3}\right)},\left\|\mathbf{H}^{\prime}\right\|_{\left.\mathbf{L}^{\infty}\left(\Omega ; \mathbf{R}^{3}\right)\right)}\right.$ denote constants, $\epsilon>0$ sufficient small.

In what follows, we estimate the next term. Using Taylor-Lagrange formula, we derive

$$
\begin{aligned}
& \int_{\Omega} \rho\left(s(\rho, \theta)-s\left(\rho^{\prime}, \theta^{\prime}\right)\right) \partial_{t} \theta^{\prime} d x \\
\leq & \int_{\Omega} \rho^{\prime}\left[\partial_{\rho} s\left(\rho^{\prime}, \theta^{\prime}\right)\left(\rho-\rho^{\prime}\right)+\partial_{\theta} s\left(\rho^{\prime}, \theta^{\prime}\right)\left(\theta-\theta^{\prime}\right)\right] \partial_{t} \theta^{\prime} d x+4 c(\cdot) \int_{\Omega} \Gamma\left(\rho, \theta \mid \rho^{\prime}, \theta^{\prime}\right) d x .(69)
\end{aligned}
$$

Similar with getting (69) and (57), respectively, we have

$$
\begin{aligned}
& -\int_{\Omega} \rho\left(s(\rho, \theta)-s\left(\rho^{\prime}, \theta^{\prime}\right)\right) \mathbf{u}^{\prime} \cdot \nabla_{x} \theta^{\prime} d x \\
\leq & -\int_{\Omega} \rho^{\prime}\left[\partial_{\rho} s\left(\rho^{\prime}, \theta^{\prime}\right)\left(\rho-\rho^{\prime}\right)+\partial_{\theta} s\left(\rho^{\prime}, \theta^{\prime}\right)\left(\theta-\theta^{\prime}\right)\right] \mathbf{u}^{\prime} \cdot \nabla_{x} \theta^{\prime} d x+c(\cdot) \int_{\Omega} \Gamma\left(\rho, \theta \mid \rho^{\prime}, \theta^{\prime}\right) d x(70)
\end{aligned}
$$

and

$$
\begin{aligned}
& \left|\int_{\Omega} \frac{\rho-\rho^{\prime}}{\rho^{\prime}} \nabla_{x} P\left(\rho^{\prime}, \theta^{\prime}\right) \cdot\left(\mathbf{u}-\mathbf{u}^{\prime}\right) d x\right| \\
\leq & c\left(\left|\nabla_{x} \rho^{\prime},\right| \nabla_{x} \theta^{\prime} \mid\right)\left(\epsilon\left\|\mathbf{u}-\mathbf{u}^{\prime}\right\|_{\mathbf{W}^{1,2}\left(\Omega ; \mathbf{R}^{3}\right)}^{2}+\int_{\Omega} \Gamma\left(\rho, \theta \mid \rho^{\prime}, \theta,\right) d x\right) .
\end{aligned}
$$


Now we estimate the last term of right-hand side of (55). By (71),

$$
\begin{aligned}
& \mid \int_{\Omega}\left(\frac{\rho-\rho^{\prime}}{\rho^{\prime}} \partial_{t} P\left(\rho^{\prime}, \theta^{\prime}\right)-\frac{\rho}{\rho^{\prime}} \mathbf{u} \cdot \nabla_{x} P\left(\rho^{\prime}, \theta^{\prime}\right)\right) d x \\
\leq & \int_{\Omega} \frac{\rho-\rho^{\prime}}{\rho^{\prime}}\left(\partial_{t} P\left(\rho^{\prime}, \theta^{\prime}\right)+\mathbf{u} \cdot \nabla_{x} P\left(\rho^{\prime}, \theta^{\prime}\right)\right) d x+\int_{\Omega} P\left(\rho^{\prime}, \theta^{\prime}\right) \mathbf{d i v}_{x} \mathbf{u} d x \\
& +c\left(\left|\nabla_{x} \rho^{\prime},\right| \nabla_{x} \theta^{\prime} \mid\right)\left(\epsilon\left\|\mathbf{u}-\mathbf{u}^{\prime}\right\|_{\mathbf{W}^{1,2}\left(\Omega ; \mathbf{R}^{3}\right)}^{2}+\int_{\Omega} \Gamma\left(\rho, \theta \mid \rho^{\prime}, \theta,\right) d x\right) .
\end{aligned}
$$

Thus by (55)-(56), (63)-(69) and (72), we obtain the following relative entropy inequality

$$
\begin{aligned}
& \int_{\Omega}\left(\frac{1}{2} \rho\left|\mathbf{u}-\mathbf{u}^{\prime}\right|^{2}+\frac{1}{2}\left|\mathbf{H}-\mathbf{H}^{\prime}\right|^{2}+\Gamma\left(\rho, \theta \mid \rho^{\prime}, \theta\right)\right)(\tau, \cdot) d x+\nu \int_{0}^{\tau} \int_{\Omega}\left|\nabla \times \mathbf{H}^{\prime}-\nabla \times \mathbf{H}\right|^{2} d x d t \\
& +\int_{0}^{\tau} \int_{\Omega}\left(\frac{\theta^{\prime}}{\theta} \mathbf{S}\left(\theta, \nabla_{x} \mathbf{u}\right): \nabla_{x} \mathbf{u}-\mathbf{S}\left(\theta^{\prime}, \nabla_{x} \mathbf{u}^{\prime}\right):\left(\nabla_{x} \mathbf{u}-\nabla_{x} \mathbf{u}^{\prime}\right)-\mathbf{S}\left(\theta, \nabla_{x} \mathbf{u}\right): \nabla_{x} \mathbf{u}^{\prime} d x d t\right) \\
& +\int_{0}^{\tau} \int_{\Omega}\left(\frac{\mathbf{q}\left(\theta, \nabla_{x} \theta\right) \cdot \nabla_{x} \theta^{\prime}}{\theta}-\frac{\theta^{\prime}}{\theta} \frac{\mathbf{q}\left(\theta, \nabla_{x} \theta\right) \cdot \nabla_{x} \theta}{\theta} d x d t\right) \\
& \leq \int_{0}^{\tau}\left(\epsilon\left\|\mathbf{u}^{\prime}-\mathbf{u}\right\|_{\mathbf{W}_{0}^{1,2}\left(\Omega ; \mathbf{R}^{3}\right)}^{2}+c^{\prime}(\epsilon, \cdot) \int_{\Omega}\left(\Gamma\left(\rho, \theta \mid \rho^{\prime}, \theta^{\prime}\right)+\frac{\rho}{2}\left|\mathbf{u}-\mathbf{u}^{\prime}\right|^{2}\right) d x\right) d t \\
& +\int_{0}^{\tau} \int_{\Omega}\left(P\left(\rho^{\prime}, \theta^{\prime}\right)-P(\rho, \theta)\right) \mathbf{d i v} \mathbf{v}_{x} \mathbf{u}^{\prime}+\left(\left(\nabla \times \mathbf{H}^{\prime}\right) \times \mathbf{H}^{\prime}\right) \cdot\left(\mathbf{u}^{\prime}-\mathbf{u}\right) d x d t \\
& +\int_{0}^{\tau}\left(\epsilon c^{\prime \prime}\left\|\mathbf{u}-\mathbf{u}^{\prime}\right\|_{\mathbf{W}^{1,2}\left(\Omega ; \mathbf{R}^{3}\right)}^{2}+c_{\epsilon} \int_{\Omega}\left|\mathbf{H}^{\prime}-\mathbf{H}\right|^{2} d x\right) d t \\
& -\int_{0}^{\tau} \int_{\Omega} \rho^{\prime}\left[\partial_{\rho} s\left(\rho^{\prime}, \theta^{\prime}\right)\left(\rho-\rho^{\prime}\right)+\partial_{\theta} s\left(\rho^{\prime}, \theta^{\prime}\right)\left(\theta-\theta^{\prime}\right)\right]\left(\partial_{t} \theta^{\prime}+\mathbf{u}^{\prime} \cdot \nabla_{x} \theta^{\prime}\right) d x d t \\
& +\int_{0}^{\tau} \int_{\Omega} \frac{\rho-\rho^{\prime}}{\rho^{\prime}}\left(\partial_{t} P\left(\rho^{\prime}, \theta^{\prime}\right)+\mathbf{u} \cdot \nabla_{x} P\left(\rho^{\prime}, \theta^{\prime}\right)\right) d x d t .
\end{aligned}
$$

Next we simplify the relative entropy inequality (73). Using (52) and the fact that $\rho^{\prime}$ and $\mathbf{u}^{\prime}$ satisfy the equation of continuity (1), we derive

$$
\begin{aligned}
& -\int_{\Omega} \rho^{\prime}\left[\partial_{\rho} s\left(\rho^{\prime}, \theta^{\prime}\right)\left(\rho-\rho^{\prime}\right)+\partial_{\theta} s\left(\rho^{\prime}, \theta^{\prime}\right)\left(\theta-\theta^{\prime}\right)\right]\left(\partial_{t} \theta^{\prime}+\mathbf{u}^{\prime} \cdot \nabla_{x} \theta^{\prime}\right) d x+\int_{\Omega} \frac{\rho-\rho^{\prime}}{\rho^{\prime}}\left(\partial_{t} P\left(\rho^{\prime}, \theta^{\prime}\right)+\mathbf{u} \cdot \nabla_{x} P\left(\rho^{\prime}, \theta^{\prime}\right)\right) d x \\
= & -\int_{\Omega} \rho^{\prime}\left(\theta-\theta^{\prime}\right)\left(\frac{1}{\theta}\left(\mathbf{S}\left(\theta^{\prime}, \nabla_{x} \mathbf{u}^{\prime}\right): \nabla_{x} \mathbf{u}^{\prime}-\frac{\mathbf{q}\left(\theta^{\prime}, \nabla_{x} \theta^{\prime}\right) \cdot \nabla_{x} \theta^{\prime}}{\theta^{\prime}}\right)-\mathbf{d i v}_{x}\left(\frac{\mathbf{q}\left(\theta^{\prime}, \nabla_{x} \theta^{\prime}\right)}{\theta}\right)\right) d x \\
& +\int_{\Omega}\left(\left(\theta-\theta^{\prime}\right) \partial_{\theta} P\left(\rho^{\prime}, \theta^{\prime}\right)+\left(\rho-\rho^{\prime}\right) \partial_{\rho} P\left(\rho^{\prime}, \theta^{\prime}\right)\right) \operatorname{div}_{x} \mathbf{u}^{\prime} d x
\end{aligned}
$$

Note that

$$
\begin{aligned}
& \left|\int_{\Omega}\left(P\left(\rho^{\prime}, \theta^{\prime}\right)-\partial_{\rho} P\left(\rho^{\prime}, \theta^{\prime}\right)\left(\rho^{\prime}-\rho\right)-\partial_{\theta} P\left(\rho^{\prime}, \theta^{\prime}\right)\left(\theta^{\prime}-\theta\right)-P(\rho, \theta) \operatorname{div}_{x} \mathbf{u}^{\prime}\right) d x\right| \\
\leq & c\left\|\operatorname{div}_{x} \mathbf{u}\right\|_{\mathbf{L}^{\infty}(\Omega)} \int_{\Omega} \Gamma\left(\rho, \theta \mid \rho^{\prime}, \theta^{\prime}\right) d x .
\end{aligned}
$$


Thus by (73)-(74) and (75), for any $\epsilon>0$, we obtain

$$
\begin{aligned}
& \int_{\Omega}\left(\frac{1}{2} \rho\left|\mathbf{u}-\mathbf{u}^{\prime}\right|^{2}+\frac{1}{2}\left|\mathbf{H}-\mathbf{H}^{\prime}\right|^{2}+\Gamma\left(\rho, \theta \mid \rho^{\prime}, \theta\right)\right)(\tau, \cdot) d x+\nu \int_{0}^{\tau} \int_{\Omega}\left|\nabla \times \mathbf{H}^{\prime}-\nabla \times \mathbf{H}\right|^{2} d x d t \\
& +\int_{0}^{\tau} \int_{\Omega}\left(\frac{\theta^{\prime}}{\theta} \mathbf{S}\left(\theta, \nabla_{x} \mathbf{u}\right): \nabla_{x} \mathbf{u}-\mathbf{S}\left(\theta^{\prime}, \nabla_{x} \mathbf{u}^{\prime}\right):\left(\nabla_{x} \mathbf{u}-\nabla_{x} \mathbf{u}^{\prime}\right)-\mathbf{S}\left(\theta, \nabla_{x} \mathbf{u}\right): \nabla_{x} \mathbf{u}^{\prime}+\frac{\theta-\theta^{\prime}}{\theta^{\prime}} \mathbf{S}\left(\theta^{\prime}, \nabla_{x} \mathbf{u}^{\prime}: \nabla_{x} \mathbf{u}^{\prime}\right) d x d t\right) \\
& +\int_{0}^{\tau} \int_{\Omega}\left(\frac{\mathbf{q}\left(\theta, \nabla_{x} \theta\right) \cdot \nabla_{x} \theta^{\prime}}{\theta}-\frac{\theta^{\prime}}{\theta} \frac{\mathbf{q}\left(\theta, \nabla_{x} \theta\right) \cdot \nabla_{x} \theta}{\theta}+\left(\theta^{\prime}-\theta\right) \frac{\mathbf{q}\left(\theta^{\prime}, \nabla_{x} \theta^{\prime}\right)}{\theta^{\prime 2}}+\frac{\mathbf{q}\left(\theta^{\prime}, \nabla_{x} \theta^{\prime}\right)}{\theta^{\prime}} \cdot \nabla_{x}\left(\theta-\theta^{\prime}\right)\right) d x d t \\
\leq & \int_{0}^{\tau}\left(\epsilon\left\|\mathbf{u}^{\prime}-\mathbf{u}\right\|_{\mathbf{W}_{0}^{1,2}\left(\Omega ; \mathbf{R}^{3}\right)}^{2}+c^{\prime}(\epsilon, \cdot) \int_{\Omega}\left(\Gamma\left(\rho, \theta \mid \rho^{\prime}, \theta^{\prime}\right)+\frac{1}{2} \rho\left|\mathbf{u}-\mathbf{u}^{\prime}\right|^{2}\right) d x\right) d t \\
& +\int_{0}^{\tau}\left(\epsilon c^{\prime \prime}\left\|\mathbf{u}-\mathbf{u}^{\prime}\right\|_{\mathbf{W}^{1,2}\left(\Omega ; \mathbf{R}^{3}\right)}^{2}+c_{\epsilon} \int_{\Omega}\left|\mathbf{H}^{\prime}-\mathbf{H}\right|^{2} d x\right) d t \\
& +\int_{\Omega}\left(P\left(\rho^{\prime}, \theta^{\prime}\right)-\partial_{\rho} P\left(\rho^{\prime}, \theta^{\prime}\right)\left(\rho^{\prime}-\rho\right)-\partial_{\theta} P\left(\rho^{\prime}, \theta^{\prime}\right)\left(\theta^{\prime}-\theta\right)-P(\rho, \theta) \mathbf{d i v}_{x} \mathbf{u}^{\prime}\right) d x \\
\leq & \int_{0}^{\tau}\left(\epsilon\left\|\mathbf{u}^{\prime}-\mathbf{u}\right\|_{\mathbf{W}_{0}^{1,2}\left(\Omega ; \mathbf{R}^{3}\right)}^{2}+c^{\prime}(\epsilon, \cdot) \int_{\Omega}\left(\Gamma\left(\rho, \theta \mid \rho^{\prime}, \theta^{\prime}\right)+\frac{1}{2} \rho\left|\mathbf{u}-\mathbf{u}^{\prime}\right|^{2}\right) d x\right) d t \\
& +\int_{0}^{\tau}\left(\epsilon c^{\prime \prime}\left\|\mathbf{u}-\mathbf{u}^{\prime}\right\|_{\mathbf{W}^{1,2}\left(\Omega ; \mathbf{R}^{3}\right)}^{2}+c_{\epsilon} \int_{\Omega}\left|\mathbf{H}^{\prime}-\mathbf{H}\right|^{2} d x\right) d t .
\end{aligned}
$$

The estimate of the terms on $\mathbf{S}$ and $\mathbf{q}$ has been founded in 10 . So we omit it. To conclude that we get

$$
\begin{aligned}
& \int_{\Omega}\left(\frac{1}{2} \rho\left|\mathbf{u}-\mathbf{u}^{\prime}\right|^{2}+\frac{1}{2}\left|\mathbf{H}-\mathbf{H}^{\prime}\right|^{2}+\Gamma\left(\rho, \theta \mid \rho^{\prime}, \theta\right)\right)(\tau, \cdot) d x+\nu \int_{0}^{\tau} \int_{\Omega}\left|\nabla \times \mathbf{H}^{\prime}-\nabla \times \mathbf{H}\right|^{2} d x d t \\
& +c_{1} \int_{0}^{\tau} \int_{\Omega}\left(\left|\nabla_{x} \mathbf{u}^{\prime}-\nabla_{x} \mathbf{u}\right|^{2}+\left|\nabla_{x} \theta^{\prime}-\nabla_{x} \theta\right|^{2}+\left|\nabla_{x} \log \theta^{\prime}-\nabla_{x} \log \theta\right|^{2}\right) d x d t \\
\leq & c_{2} \int_{0}^{\tau} \int_{\Omega}\left(\Gamma\left(\rho, \theta \mid \rho^{\prime}, \theta^{\prime}\right)+\frac{1}{2} \rho\left|\mathbf{u}-\mathbf{u}^{\prime}\right|^{2}\right) d x d t \\
& +\int_{0}^{\tau}\left(\epsilon c^{\prime \prime}\left\|\mathbf{u}-\mathbf{u}^{\prime}\right\|_{\mathbf{W}^{1,2}\left(\Omega ; \mathbf{R}^{3}\right)}^{2}+c_{\epsilon} \int_{\Omega}\left|\mathbf{H}^{\prime}-\mathbf{H}\right|^{2} d x\right) d t, \text { for almost all } \tau \in(0, T) .
\end{aligned}
$$

Furthermore, for sufficient small $\epsilon>0$, we obtain

$$
\begin{aligned}
& \int_{\Omega}\left(\frac{1}{2} \rho\left|\mathbf{u}-\mathbf{u}^{\prime}\right|^{2}+\frac{1}{2}\left|\mathbf{H}-\mathbf{H}^{\prime}\right|^{2}+\Gamma\left(\rho, \theta \mid \rho^{\prime}, \theta\right)\right)(\tau, \cdot) d x+\nu \int_{0}^{\tau} \int_{\Omega}\left|\nabla \times \mathbf{H}^{\prime}-\nabla \times \mathbf{H}\right|^{2} d x d t \\
& +\left(c_{1}-c^{\prime \prime} \epsilon\right) \int_{0}^{\tau} \int_{\Omega}\left(\left|\nabla_{x} \mathbf{u}^{\prime}-\nabla_{x} \mathbf{u}\right|^{2}+\left|\nabla_{x} \theta^{\prime}-\nabla_{x} \theta\right|^{2}+\left|\nabla_{x} \log \theta^{\prime}-\nabla_{x} \log \theta\right|^{2}\right) d x d t \\
\leq & c_{2} \int_{0}^{\tau}\left(\int_{\Omega}\left(\Gamma\left(\rho, \theta \mid \rho^{\prime}, \theta^{\prime}\right)+\frac{1}{2} \rho\left|\mathbf{u}-\mathbf{u}^{\prime}\right|^{2}+\frac{1}{2}\left|\mathbf{H}^{\prime}-\mathbf{H}\right|^{2}\right)\right) d x
\end{aligned}
$$

which implies that

$$
\rho \equiv \rho^{\prime}, \quad \mathbf{u} \equiv \mathbf{u}^{\prime}, \quad \theta \equiv \theta^{\prime}, \quad \mathbf{H} \equiv \mathbf{H}^{\prime} .
$$

This completes the proof. 
Acknowledgements The author also expresses his sincere thanks to the anonymous referees for very careful reading and for providing many valuable comments and suggestions which led to improvement of this paper. The author expresses his sincerely thanks to prof Zhifei Zhang and prof Yong Li for their comments and suggestions. The author is supported by NSFC No 11201172, 11071101, the post doctor fund 2012M510243, SRFDP Grant No 20120061120002 and the 985 Project of Jilin University.

\section{References}

1. Carrillo J., Jüngel A., Markowich P.A., Toscani G., Unterreiter A., Entropy dissipationmethods for degenerate parabolic problems and generalized Sobolev inequalities, Monatshefte Math., 2001, 133, 1-82

2. Chemin J.-Y., About weak-strong uniqueness for the 3D incompressible Navier-Stokes system, Comm. Pure. Appl. Math., 2011, 64, 1587-1598

3. Dafermos C.M., The second law of thermodynamics and stability, Arch. Ration. Mech. Anal., 1979, 70, 167-179

4. Ducomet B., Feireisl E., The equations of magnetohydrodynamics: on the interaction between matter and radiation in the evolution of gaseous stars, Comm. Math. Phys., 2006, 266, 595-629

5. Eliezer S., Ghatak A., Hora H., An Introduction to Equations of States, Theory and Applications, Cambridge University Press, Cambridge, 1986

6. Feireisl E., Dynamics of Viscous Compressible Fluids. Oxford University Press, Oxford, 2004

7. Feireisl E., Novotný A., Singular Limits in Thermodynamics of Viscous Fluids, Birkhäuser-Verlag, Basel, 2009

8. Feireisl E., Novotný A., Sun Y.Z., Suitable weak solutions to the Navier-Stokes equations of compressible viscous fluids, Indiana Univ. Math. J., 2011, 60, 611-632

9. Feireisl E., Jin B.J., Novotný A., Relative entropies, suitable weak solutions, and weakstrong uniqueness for the compressible Navier-Stokes system, J. Math. Fluid Mech., 2012, 14, 717-730

10. Feireisl E., Novotný A., Weak-strong uniqueness property for the full Navier-StokesFourier system, Arch. Rational Mech. Anal., 2012, 204, 683-706

11. Hu X., Wang D., Global solutions to the three dimensional full compressible magnetohydrodynamic flows, Comm. Math. Phys., 2008, 283, 255-284

12. Jiang S., Ju Q.C., Li F.C., Incompressible limit of the compressible Magnetohydrodynamic equations with periodic boundary conditions, Comm. Math. Phys., 2010, 297, 371-400

13. Klein R., Botta N., Schneider T., Munz C.D., Roller S., Meister A., Hoffmann L., Sonar T., Asymptotic adaptive methods for multi-scale problems in fluid mechanics, J. Engrg. Math., 2001, 39, 261-343

14. Kwon Y.S., Trivisa K., On the incompressible limits for the full magnetohydrodynamics flows, J. Diff. Eqns., 2011, 251, 1990-2023

15. Ladyzhenskaya O.A., Themathematical Theory of Viscous Incompressible Flow, Gordon and Breach, New York, 1969

16. Leray J., Sur le mouvement d'un liquide visqueux emplissant l'espace, Acta Math., 1934, $63,193-248$

17. Lions P.-L., Mathematical topics in fluid dynamics. Compressible Model, vol. 2. Oxford Science Publication, Oxford, 1998

18. Germain P., Strong solutions and weak-strong uniqueness for the nonhomogeneous Navier-Stokes system, J. Anal. Math., 2008, 105, 169-196

19. Germain P., Weak-strong uniqueness for the isentropic compressible Navier-Stokes system, J. Math. Fluid Mech., 2011, 13, 169-196

20. Saint-Raymond L., Hydrodynamic limits: some improvements of the relative entropy method, Ann. I. H. Poincar AN., 2009, 26, 137-146

21. Serrin J., On the interior regularity of weak solutions of the Navier-Stokes equations, Arch. Ration. Mech. Anal., 1962, 9, 187-195

22. Temam R., Navier-Stokes Equations, North-Holland, Amsterdam, 1977 